\title{
A case report of necrotizing sialometaplasia with immunohisto- logical findings and a review of the literature
}

\author{
Yutaka Kimura $^{1}$, Kenichi Matsuzaka ${ }^{1}$, Kaichi Matsuoka ${ }^{1}$, Takashi Muramatsu², Yoko Yokoyama ${ }^{3}$, \\ Takashi Inoue ${ }^{1}$ \\ ${ }^{1}$ Department of Clinical Pathophysiology, Tokyo Dental College, Chiba, Japan \\ ${ }^{2}$ Department of Pathology, Tokyo Dental College, Chiba, Japan \\ ${ }^{3}$ Department of Clinical Oral Health Science, Division of Oral and Maxillofacial Surgery, Tokyo Dental College, Tokyo, Japan
}

Abstract: We report a case of necrotizing sialometaplasia arising as bilateral ulcers in the palate of a 20 -year-old Japanese female patient. Based on histopathological and immunohistochemical findings, we discuss how to distinguish the condition from neoplastic lesions such as squamous cell carcinoma or mucoepidermoid carcinoma. Histopathologically, the ulcer bed of granulation tissue contained hyperplastic capillary vessels, inflammatory cell infiltrates, necrotizing and degenerating palatal glands, and squamous metaplasia of ducts and acini leaving lobular structures of the mucous gland. Immunohistochemically, the epithelial islands were positive for pancytokeratin (CK), CK7 and S-100 protein, partially positive for a-smooth muscle actin, and not positive for periodic acid-Schiff reaction. We diagnosed the lesion as a necrotizing sialometaplasia. It healed spontaneously without treatment, and there was no recurrence. [Oral Med Pathol 2011; 15: 87-90 doi: 10.3353/omp.15.87]

Key words: bilateral ulcer, cytokeratin 7, literature review, necrotizing sialometaplasia, palate

Correspondence: Kenichi Matsuzaka, Department of Clinical Pathophysiology, Tokyo Dental College, 1-2-2 Masago, Mihama-ku, Chiba, 261-8502, Japan Phone: +81-43-270-3581, Fax: +81-43-270-3583, E-mail: matsuzak@tdc.ac.jp

\section{Introduction}

Necrotizing sialometaplasia (NS) was first reported by Abrams et al. in 1973 (1). It is a relatively rare and benign condition of salivary gland metaplasia with necrosis and inflammation. The importance of this disease is that it resembles a malignant tumor both histopathologically and clinically, and the possibility of an incorrect diagnosis exists. Therefore, we should take NS into account when making a diagnosis. In this paper, a case of NS on both sides of the palate in a 20 -year-old female is immunohistologically characterized and described.

\section{Case Report}

A 20-year-old Japanese female was admitted to the Department of Oral and Maxillofacial Surgery, Tokyo Dental College Suidobashi Hospital, with a chief complaint of a painful ulcer about $15 \times 10 \mathrm{~mm}$ existing in her bilateral palate (Fig. 1a). The patient had a history of asthma. Two weeks before being admitted to the hospital, she had been conscious of a slight spontaneous pain and swelling in her palate, and had consulted an otolaryngologist, who prescribed a vitamin preparation. However, she then consulted a dentist because her symptoms had not improved.
At the initial visit, she received a blood test, a microorganism test, a histopathological test, and an X-ray examination; in addition, the wound was disinfected. There were no specific radiographic findings. At the second visit, she underwent a CT scan and the wound was disinfected again. Some bone resorption was evident in the CT image (Fig. 1b), and follow-up care was given. The histopathological diagnosis was reported as a NS. The reductive appearance of the ulcer on the palate is shown in Fig. 1c. This case of NS healed without treatment, and there was no recurrence.

\section{Histopathological and immunohistochemical findings}

The resected tissue was fixed in $20 \%$ neutral buffered formalin, embedded in paraffin, and processed for routine histological examination. Microscopically, fibrous connective tissue was covered with a parakeratotic stratified squamous epithelium with extensions of the epithelial legs. The epithelium had become ulcerated. The submucosa contained granulation tissue hyperplasia of capillary vessels, inflammatory cell infiltration such as neutrophils and lymph cells, necrotizing and degenerating palatine glands with mucus spillage and dissolution of the acinar structure, squamous metaplasia of ducts, and acini with preserved lobular structures (Figs. 2a \& 2b). In addition, partially 

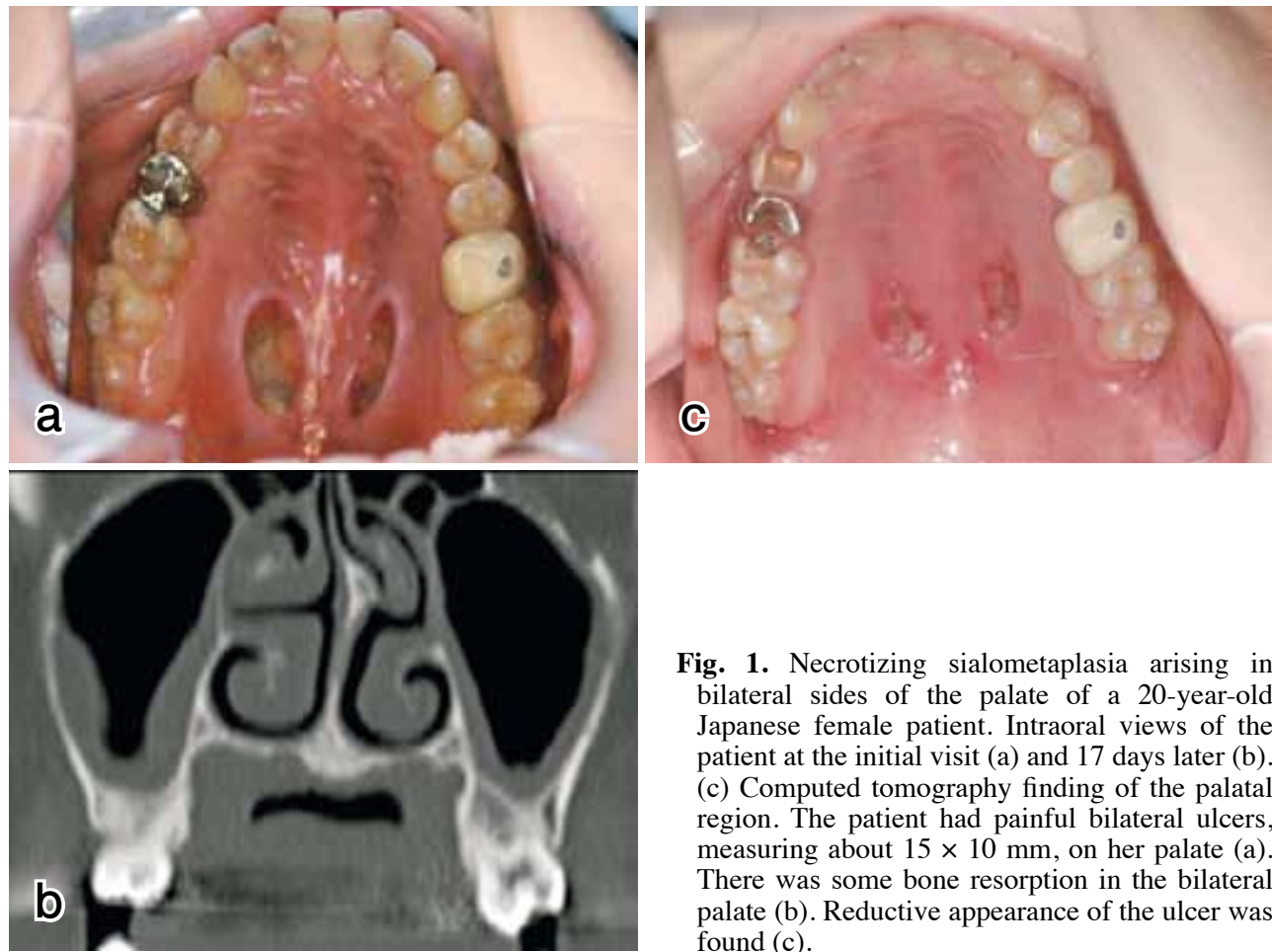

Fig. 1. Necrotizing sialometaplasia arising in bilateral sides of the palate of a 20 -year-old Japanese female patient. Intraoral views of the patient at the initial visit (a) and 17 days later (b)

(c) Computed tomography finding of the palatal region. The patient had painful bilateral ulcers, measuring about $15 \times 10 \mathrm{~mm}$, on her palate (a). There was some bone resorption in the bilateral palate (b). Reductive appearance of the ulcer was found (c).

assembled neutrophils were observed. Squamous cells with weak nuclear atipia were seen in some areas; however, almost all areas displayed a bland, benign appearance of the squamous cells. The contents of salivary ducts and tissue surrounding the destroyed ducts were positive for periodic acid-Schiff reaction (Fig. 2c). Mucus seemed to be flowing from the ducts. Immunohistochemically, the metaplastic epithelial islands and the salivary ducts were positive for pan-cytokeratin (Fig. 2d), S-100 protein, and cytokeratin7 (CK7) (Fig. 2e). Ducts and luminal trace parts of metaplastic epithelial islands were positive for $\alpha$-smooth muscle actin $(\alpha$-SMA).
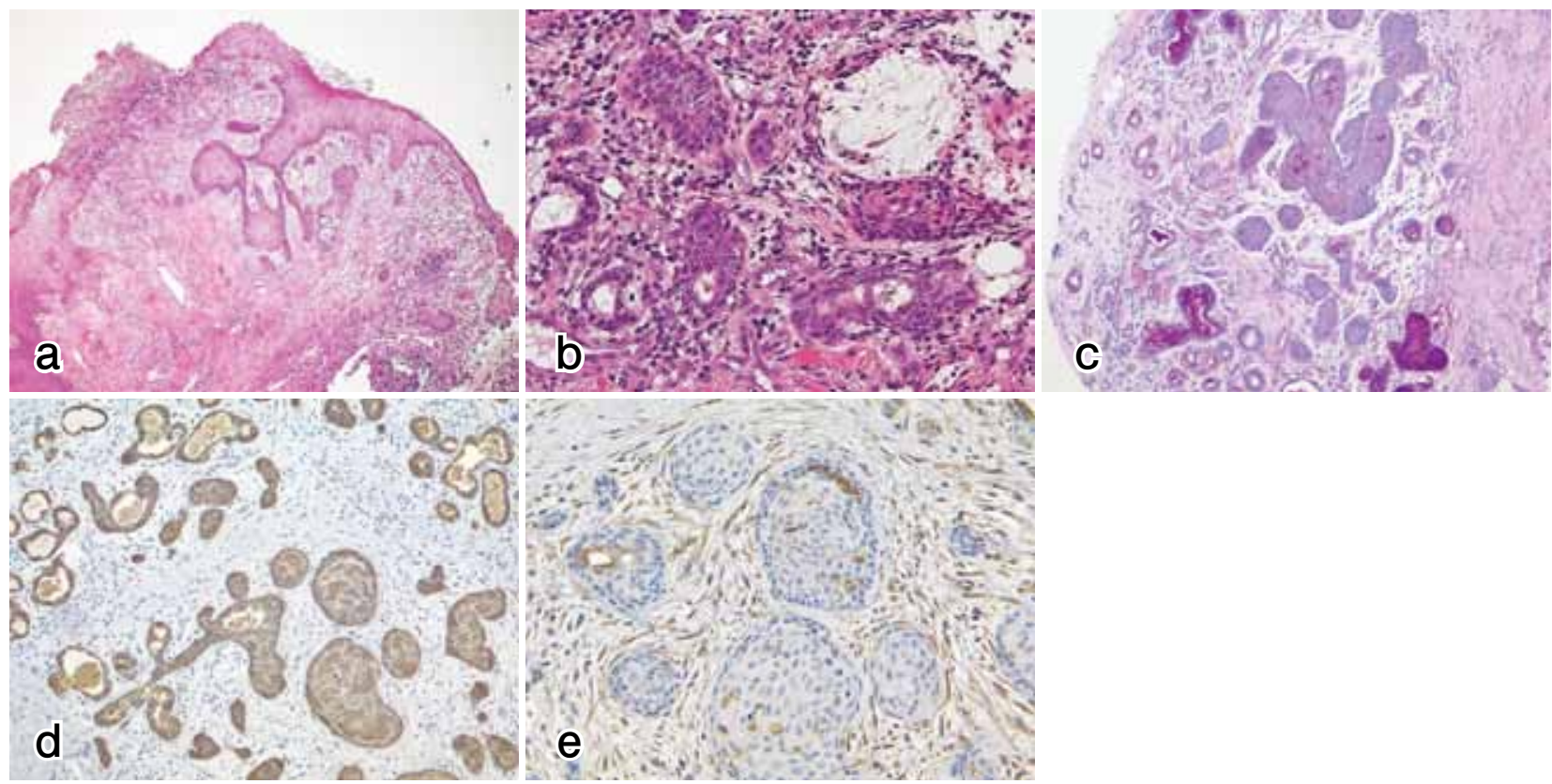

Fig. 2. Histopathology of necrotizing sialometaplasia. (a, b) Hematoxylin and eosin stain; (c) periodic acid-Schiff (PAS) stain immunoperoxidase stain for pan-cytokeratin (d) and cytokeratin 7 (CK7) (e). (a) $\times 40$; (b, e) $\times 200$; (c-d) $\times 100$. The mucosa was partially ulcerated. The lamina propria space was replaced with fribrous granulation tissue, in which inflammatory cell infiltrates and squamous epithelial nests were pronounced (a). The epithelial nests were metaplastic changes of salivary ducts, around which necrotic or degenerative glandular lobules were observed (b). PAS-positive amorphous materials were retained in the salivary duct lumen and scattered around deformed or destroyed ducts (c). Most of the epithelial cells forming both the metaplastic epithelial islands and salivary ducts were positive for pancytokeratin (d), while CK7 was definitely positive in luminal cells of the ducts and faintly and occasionally positive in lumen-like traces within the metaplastic epithelial islands (e). 


\section{Discussion}

It is said that NS is cured spontaneously within 6 to 12 weeks, and there is no need for surgery (2). However, since NS resembles a malignant tumor histopathologically and clinically (2-3), it is possible that it could be misdiagnosed as a squamous cell carcinoma (SCC) or a mucoepidermoid carcinoma (MEC). Therefore, care is needed to make an accurate diagnosis. NS is a relatively rare disease found in all age groups. Mesa et al. reported that it represents only $0.03 \%$ of all lesions biopsied during a 10-year period (4). Brannon et al. reported that the average age of a patient with NS is 45.9 years, and that NS occurs in men 1.86 times as frequently as women (2). Oda et al. reported an average age of 44.1 years and no gender gap in Japan (5). The most frequent site of NS is the palate, which accounts for $70 \%$ to $80 \%$ of all cases $(2,6)$. However, bilateral symmetrical affection occurs rarely and has been reported as a $12 \%$ incidence (2). Other sites reported include the parotid gland $(2,7)$, nasal cavity $(2,8-9)$, upper pharynx (10), gingiva (11), lip (2, 12-13), buccal mucosa (14-15), back of the molar $(2,11,16)$, tongue $(2,17)$ and maxillary sinus $(18)$.

The clinical finding of NS is the formation of an ulcer and a mass. The boundary of the undermined ulcer is comparatively clear. It has been reported that the size ranges from $0.5 \mathrm{~cm}$ to $4 \mathrm{~cm}$, with an average of $2 \mathrm{~cm} \mathrm{(2),} \mathrm{and} \mathrm{that}$ erythema is sometimes observed surrounding the ulcer (1). Oppressive or dull pain is noted in many cases. However, slight pain is often a clinical finding, and in some cases patients report a numb feeling. In one investigation, the authors thought that the numb feeling was caused by several factors, such as the influence of the greater palatine nerve surrounding the inflammatory stimulus and hematogenous disorder, infarction, and necrosis of the gland tissue (19).

There are various opinions about the cause of NS. The gland infarction theory proposed by Arguelles et al., in which NS originates as a hematogenous disorder due to various causes is the most supported (20). The ischemic necrosis and the ulcer formation of the saliva gland are caused by the squamous metaplasia of glands regenerating epithelial tissue during the reparative process. NS was studied in short-term experiments by Standish et al. (21) and
Englander et al. (22), who ligated blood vessels of rat mandibular glands. They suggested that an ischemia change is related to the occurrence of NS. Smoking, chronic alcohol use, unsuitable dentures, local anesthesia, surgical treatment, external wounds, upper respiratory infections, tumors, operations, irradiation and frequent emesis are thought to be local causes of NS (2, 8, 23-26). Dominguez-Malagon et al. (24) and Yoshioka et al. (25) reported that T-cell lymphoma is considered as a causative condition of NS. Ylikontiola $e t$ al. reported that a girl's adenoidectomy may have affected branches of the palatine arteries in the soft palate, compromised the underlying vascular supply and likely caused infarction of the salivary gland tissue (26). In our case, the possible causes described above were not found. Disturbances of nutrition caused by eating disorder, hyperpiesia, diabetes or tuberculosis are thought to be whole body incitement $(1,19,27-28)$.

The features of NS described by Abrams et al. include the following: i) lobular infarction or necrosis; ii) blandappearing nuclear morphology of the squamous cells; iii) simultaneous metaplasia of ducts and mucous acini; iv) prominent granulation tissue and inflammatory components; and v) maintenance of the general lobular morphology in spite of fairly extensive inflammatory and metaplastic changes often involving more than one lobule (1). In the present case, necrotic and degenerating palatine glands and a bland-appearing nuclear morphology of the squamous cells were observed. Part of the lobular morphology was indistinct, but most of the cells preserved a lobular architecture. The preservation of normal lobular architecture of salivary glands seems to be a key feature that distinguishes NS from neoplasia (1). Anneroth and Hansen clarified the histopathogenesis of NS by suggesting it has five histopathologic stages: i) infarction, ii) sequestration, iii) ulceration, iv) reparation, and v) healed. Our case is considered to be at the ulceration or reparation stage (6).

The results of immunohistochemical findings have been reported (Table 1). Rizkalla et al. investigated the use of immunohistochemistry to distinguish NS from SCC and MEC by identifying residual myoepithelial cells and cytokeratin expression (29). Immunohistochemical staining showed that residual myoepithelial cells and CK7 expression

Table 1. Histochemical and immunohistochemical profiles of necrotizing sialometapasia documented in the literature

\begin{tabular}{|c|c|c|c|c|c|}
\hline \multirow{2}{*}{ staining } & \multicolumn{4}{|c|}{ reports by (reference \#) } & \multirow{2}{*}{ present case } \\
\hline & Oda Y et al.(5) & Miki Y et al. (19) & Rizkalla H et al.(29) & Lee DJ et al. (30) & \\
\hline periodic acid schiff (PAS) & $(+)$ & $(+)$ & & & $(+)$ \\
\hline pan-cytokeratin & & $(+)$ & & & $(+)$ \\
\hline cytokeratin 5,6 & & & $(+)$ & $(+)$ & \\
\hline cytokeratin 7 & & & $(+)$ & $(+)$ & $(+)$ \\
\hline cytokeratin 7, 8 (CAM 5.2) & & & $(+)$ & & \\
\hline cytokeratin 18 & & $(+)$ & & & \\
\hline$\alpha$-smooth muscle actin & & & $(+)$ & $(+)$ & $( \pm)$ \\
\hline calponin & & & $(+)$ & & \\
\hline S-100 protein & $(-)$ & & $(+)$ & & $(+)$ \\
\hline p63 & & & $(+)$ & $(+)$ & \\
\hline Ki-67 & & $( \pm)$ & & & \\
\hline
\end{tabular}


may help to distinguish NS from similar conditions. In general, squamous metaplasia is considered to occur in acinar and ductal components (23-24). S-100 protein and $\alpha$-SMA, well known as myoepithelial cell a markers, were found to be positive in solid epithelial islands, which meant they were myoepithelial characters. The solid epithelial islands were also considered to simultaneously obtain ductal characteristics, because moderate CK7 expression was occasionally found in the lumen of the epthelial islands with duct-like structures and the solid epithelial islands. These results indicated their epithelium metaplastic nature from salivary gland terminal cells. Considering the staining results, we diagnosed this NS from an ulcer in the bilateral palate.

In summary, the ability to distinguish NS from SCC and MEC relies on characterized morphological features. While hematoxylin-eosin staining remains the gold standard for making a diagnosis, the use of immunohistochemical staining to detect residual myoepithelial cells and CK7 expression is helpful as an adjunct in difficult cases. As shown in the case reported here, it is important to distinguish NS from malignant tumors and to employ treatment methods based on histopathological diagnosis at an early stage to avoid overtreatment.

\section{References}

1. Abrams AM, Melrose RJ, Howell FV. Necrotizing sialometaplasia: A disease simulating malignancy. Cancer 1973; 32: 130-5.

2. Brannon RB, Fowler CB, Hartman KS. Necrotizing sialometaplasia: A clinicopathologic study of sixty-nine cases and review of the literature. Oral Surg Oral Med Oral Pathol 1991; 72: 317-25.

3. WHO classification. Path Res Pract 1992; 188: 836-46.

4. Mesa ML, Gertler RS, Schneider LC. Necrotizing sialometaplasia: frequency of histologic misdiagnosis. Oral Surg Oral Med Oral Pathol 1984; 57: 71-3.

5. Oda Y, Komiyama K, Isoda J, et al. A case of necrotizing sialometaplasia of the palate. Nihon Univ Dent J 1999; 73: 665-70.

6. Anneroth G, Hansen LS. Necrotizing sialometaplasia: the relationship of its pathogenesis to its clinical characteristics. Int J Oral Surg 1982; 11: 283-91.

7. Batsakis JG. Sialometaplasia. Arch Otolaryngol 1976; 102: 191.

8. Maisel RH, Johnson WH, Anderson HA, Cantrell RW. Necrotizing sialometaplasia involving the nasal cavity. Laryngoscope 1977; 87: 429-34.

9. Chen KT. Necrotizing sialometaplasia of the nasal cavity. Am J Otolaryngol 1982; 3: 444-6.

10. Merwin GE, Duckert LG, Pollak K. Necrotizing sialometaplasia of the nasopharynx. Ann Otol Rhinol Laryngol 1979; 88: 348-51.

11. Forney SK, Foley JM, Sugg WE Jr, Oatis GW Jr. Necrotizing sialometaplasia of the mandible. Oral Surg Oral Med Oral Pathol 1977; 43: 720-6.
12. Matilla A, Flores T, Nogales FFJr, Galera H. Necrotizing sialometaplasia affecting the minor labial glands. Oral Surg Oral Med Oral Pathol 1979; 47:161-3.

13. Gad A, Willen H, Willen R, Thorstensson S, Ekman L. Necrotizing sialometaplasia of the lip simulating squamous cell carcinoma. Histopathology 1980; 4: 111-21.

14. Papanayotou PH, Kayavis JG, Epivatianos AA, Trigonidis G. Necrotizing sialometaplasia of the cheek: report of case and review of literature. J Oral Surg 1980; 38: 538-40.

15. Willen H, Willen R, Ekman L. Necrotizing sialometaplasia of the bucca. Acta Pathol Microbiol Scand A 1981; 89: 199-201.

16. Anneroth G, Bystedt H, Hammarstrom L. Necrotizing sialometaplasia-a malignancy simulating oral lesion. Swed Dent J 1986; 10: 53-8.

17. Poulson TC, Greer RO, Ryser RW. Necrotizing sialometaplasia obscuring an underlying malignancy: Report of a case. J Oral Maxillofac Surg 1984; 44: 570-74.

18. Johnston WH. Necrotizing sialometaplasia involving the mucous glands of the nasal cavity. Hum Pathol 1977; 8: 589-92.

19. Miki Y, Tsuruta T, Ohki H, Matsumoto M, Tanaka H, Komiyama K. A case of necrotizing sialometaplasia in Palate. J Jpn Oral Muco Membr 2004; 10: 23-9 (in Japanese).

20. Arguelles MT, Viloria JB, Talens MC, McCrory TP. Necrotizing sialometaplasia. Oral Surg Oral Med Oral Pathol 1976; 42: 86-90.

21. Standish SM, Shafer WG. Serial histologic effects of rat submaxillary and sublingual salivary gland duct and blood vessel ligation. J Dent Res 1957; 36: 866-79.

22. Englander A, Cataldo E. Experimental carcinogenesis in duct-artery ligated rat submandibular gland. J Dent Res 1976; 55: 229-34.

23. Schoning H, Emshoff R. Necrotizing sialometaplasia in two patients with bulmia and chronic vomiting. Int J Oral Maxillofac Surg 1998; 27: 463-5.

24. Dominguez-Malagon H, Mosqueda-Taylor A, CanoValdez AM. Ann Diag Pathol 2009; 13: 60-4.

25. Yoshioka T, Harada M, Umekita Y, et al. Necrotizing sialometaplasia of the parotid gland associated with angiocentric T-cell lymphoma: A case report and review of the literature. Pathol Int 2010; 60: 326-9.

26. Ylikontiola L, Siponen M, Salo T, George KBS. Sialometaplasia of the soft palate in a 2-year-old girl. J Can Dent Assoc 2007; 73: 38-40.

27. SamitAM, Mashberg A, Greene GW Jr. Necrotizing sialometaplasia. J Oral Surg 1979; 37: 353-6.

28. Marciani RD, Sabes WR. Necrotizing sialometaplasia: report of three cases. J Oral Surg 1976; 34: 722-6.

29. Rizkalla H, Toner M. Necrotizing sialometaplasia versus invasive carcinoma of the head and neck: the use of myoepithelial markers and keratin subtypes as an adjunct to diagnosis. Histopathology 2007; 51: 184-9.

30. Lee DJ, Ahn HK, Koh ES, Rho YS, Chu HR. Necrotizing sialometaplasia accompanied by adenoid cystic carcinoma on the soft palate. Clin Exp Otorhinolaryngol 2009; 2: 4851 . 